\title{
Modelo Inclusivo de Desenvolvimento e Avaliação de Objetos de Aprendizagem Acessíveis voltados para o Ensino Superior em Computação.
}

\author{
Andreza Bastos Mourão ${ }^{1,2}$, José Francisco Magalhães Netto ${ }^{1}$ \\ ${ }^{1}$ Programa de Pós-Graduação em Informática (PPGI) - Universidade Federal do \\ Amazonas (UFAM) \\ Av. Gen. Rodrigo Otávio, Coroado. Manaus - AM - Brasil \\ ${ }^{2}$ Universidade do Estado do Amazonas (UEA) \\ Av. Darcy Vargas, 1200. Manaus - AM - Brasil. \\ \{andreza.mourao, jnetto\} @icomp.ufam.edu.br, amourao@uea.edu.br
}

\begin{abstract}
This article presents a model of development Object Learning Affordable developed by students of undergraduate education Course Degree in Computer from the University of the State of Amazonas, motivated by social inclusion of of Students with Special Educational Needs - PNEEs. The process it's divided in three stages: planning, development and validation, that allow this way, the management and monitoring of Learning Objects Affordable constructed in accordance with the standards XML, SCORM, ADDIE model and accessibility guidelines. The critical and improvements conducted by experts contributed significantly to the completion of the objects.
\end{abstract}

Resumo. Este artigo apresenta um Modelo de desenvolvimento de Objetos de Aprendizagem Acessiveis desenvolvidos por alunos de ensino superior do Curso de Licenciatura em Informática da Universidade do Estado do Amazonas, motivados pela inclusão social de alunos Portadores de Necessidades Educacionais Especiais - PNEEs. O processo está dividido em três etapas: planejamento, desenvolvimento e validação, que permitem desta forma, o gerenciamento e monitoramento dos Objetos de Aprendizagem Acessíveis construídos, em conformidade com os padrões XML, SCORM, modelo ADDIE e diretrizes de acessibilidade. As críticas e melhorias realizadas por especialistas contribuíram significativamente para a finalização dos objetos.

\section{Introdução}

Conforme a Lei 7.853 (2016), em seu artigo $1^{\circ}$ ficam estabelecidas normas gerais que asseguram o pleno exercício dos direitos individuais e sociais das pessoas portadoras de deficiências, e sua efetiva integração social. No artigo $2^{\circ}$ o estado deve garantir as pessoas com deficiência o cumprimento das disposições constitucionais e legais, inclusive dos direitos a educação. Neste sentido, é fundamental que instituições públicas e privadas realizem iniciativas, invistam e desenvolvam projetos voltados para inclusão educacional de pessoas com deficiência desde o ensino básico até o superior.

As universidades brasileiras precisam se adequar no âmbito de infraestrutura e tecnologia, visando atender uma demanda recente de alunos Portadores de Necessidades 
V Congresso Brasileiro de Informática na Educação (CBIE 2016)

Anais do XXVII Simpósio Brasileiro de Informática na Educação (SBIE 2016)

Educacionais Especiais (PNEEs). A falta de treinamento e qualificação docente, visando atender esta demanda, apresenta-se como um fator que atinge todos os níveis de ensino e instituições públicas e privadas. É necessário um incentivo maior por parte dos governantes e gestores das instituições, com o intuito de atender a Lei Nacional de Inclusão social e digital.

A tecnologia vem sendo uma aliada importante do processo de ensino aprendizagem, sua característica principal é servir como instrumento facilitador. Recentemente, temos no Mercado uma gama de recursos educacionais como softwares, hardwares, plataformas, ambientes e linguagens voltadas para construir o ensino, intensificando assim a colaboração, inclusão e mediação a serem exploradas pelos professores.

Segundo Araujo et al (2013), a informática na educação apresenta-se como uma área promissora para facilitação do processo de ensino aprendizagem nos diversos campos do conhecimento. Os acadêmicos de Licenciatura em Informática da UEA, possuem o perfil de educador aliado ao conhecimento tecnológico, e buscam inovar suas práticas educacionais, por meio do uso e aplicação de recursos educacionais multimídias, ferramentas de autoria, ambientes, plataformas e linguagens colaborativas os quais permitam o desenvolvimento de formatos e meios diferenciados de aprender. Desta forma, surgiu a ideia de implementar um projeto voltado para alunos PNEEs, na disciplina de Tópicos Avançados na Educação com o intuito de produzir recursos educacionais inclusivos.

Este trabalho tem como objetivo apresentar um Modelo de Desenvolvimento e Avaliação de Objetos de Aprendizagem Acessíveis - OAAs, em conformidade com os padrões e diretrizes de acessibilidade, e utilizando técnicas, métodos e ferramentas voltadas para este fim. Este projeto, foi divido em três etapas importantes, tais como: planejamento, desenvolvimento e validação, e abordam conteúdos do ensino superior da área da computação.

Este artigo está organizado da seguinte forma: após a atual seção de introdução, a seção 2 descreve a metodologia científica adotada, a seção 3 descreve o modelo e suas etapas: de planejamento, desenvolvimento e a validação. Na seção 4 são descritos e discutidos os resultados apresentados e por fim na seção 5 são relatadas as considerações finais e trabalhos futuros.

\section{Metodologia Científica}

A metodologia utilizada para o desenvolvimento do projeto foi especificada em três etapas, conforme descrito a seguir:

1 - Planejamento: etapa responsável pela definição da abordagem pedagógica, elaboração do plano de aula, do cronograma do projeto e do design pedagógico.

2 - Desenvolvimento: etapa responsável pela definição do modelo a ser utilizado e da ferramenta de desenvolvimento dos objetos de aprendizagem acessíveis. Considerando os padrões e diretrizes de acessibilidade.

3 - Validação: etapa responsável pelas avaliações: avaliação da aprendizagem, de ensino, de acessibilidade e de usabilidade a ser realizada com especialistas da area pedagógica, de usabilidade e de informática na educação. 
Após a especificação das atividades, realiza-se a divisão das equipes no laboratório de informática e a apresentação das ferramentas de desenvolvimento e equipamentos a serem utilizados. O cronograma do projeto é apresentado, assim como as atividades, os procedimentos e as estratégias a serem seguidas, visando o cumprimento de inicio e encerramento do projeto. Ao final da produção dos OAAs são aplicados questionários responsáveis pela obtenção dos dados quantitativos e a geração de dados qualitativos significativos para a melhoria dos objetos.

\section{Modelo de Desenvolvimento e Avaliação de Objetos de Aprendizagem Acessíveis}

\subsection{Planejamento}

Nesta etapa a abordagem pedagógica é definida. Para este projeto adotou-se o modelo interacionista, onde o sujeito conhece o mundo por meio da interação com os objetos de conhecimento. A perspectiva epistemológica do interacionismo, representada pelo pensamento de Piaget, é uma síntese do empirismo e do racionalismo (DARSIE,1999).

Definida a abordagem elabora-se o plano de aula, onde são definidos itens como: curso, disciplina, carga horária e período. E tópicos descritivos relacionados com o objeto a ser desenvolvido, tais como: o tema ou unidade instructional, o objetivo geral e especificos da aula, a duração, o conteúdo programático, a metodologia, a forma de avaliação e a referência bibliográfica. A seguir, desenvolve-se o cronograma de atividades com datas e prazos de entrega, desenvolvimento, testes e avaliações do projeto em conformidade com o período regular do curso, e considerando os requisitos de acessibilidade do projeto. A acessibilidade deve ser considerada desde o início do projeto de criação de um objeto de aprendizagem, e não uma adaptação posterior à sua criação (MACEDO, 2010).

A etapa do Design pedagógico contou com o uso da técnica de desenvolvimento de Storyboard desenvolvido em conformidade com o plano de ensino, visando criar cenas do processo de ensino e aprendizagem estabelecido. Os storyboards segundo ABC (2016) são utilizados para o planejamento visual das cenas a serem filmadas e também para transmitir a toda a equipe o que se espera em cada cena, eles consistem em uma sequência de quadros. Cada um dos desenhos pode ser acompanhado de anotações sobre a cena, tais como: a descrição da ação, do movimento, o som (ou sons) que a acompanharão, ou qualquer outra informação que se julgar importante. Os Storyboards são desenhos rápidos e com poucos detalhes, sendo o mais objetivo possível.

O uso da técnica de criação de Storyboard permite ao aluno obter uma visão do projeto e da implementação em paralelo com o conteúdo a ser desenvolvido e o conhecimento que deve ser transmitido. Para o desenvolvimento das cenas e quadros, e das avaliações, os alunos realizam consultas com os docentes especialistas da área. E estes contribuem com os objetivos e estratégias educacionais, permitindo assim clareza na definição da sequencia instrucional e no nível cognitivo requerido nas atividades. A figura 1 abaixo, representa o modelo navegacional das cenas, criadas na ferramenta StoryboardThat (2016), disponível na web em edição free, permitindo a professores e alunos criarem projetos escolares, além de disponibilizar uma gama de ferramentas e cenários gráficos, estimulando a criatividade e o desenvolvimento de projetos 
V Congresso Brasileiro de Informática na Educação (CBIE 2016)

Anais do XXVII Simpósio Brasileiro de Informática na Educação (SBIE 2016)

educacionais, graças aos inúmeros recursos oferecidos. O Storyboard apresentado na figura 1, refere-se a disciplina de Lógica de Programação, cujo conteúdo é Algoritmos (Descrição Narrativa, Fluxograma e Portugol).

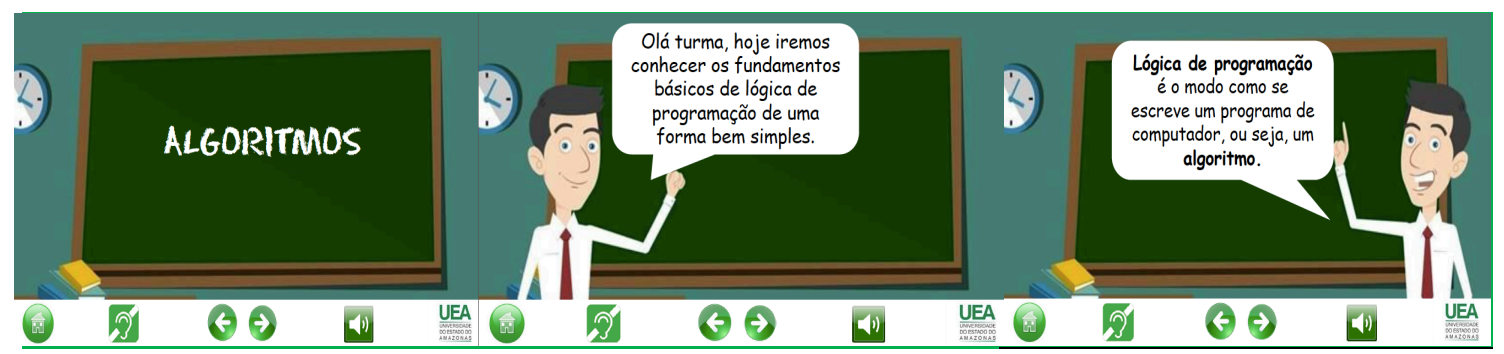

Figura 1 - Storyboard do Objeto de Aprendizagem Acessível: Algoritmos.

O Storyboard desenvolvido é apresentado às outras equipes para obter críticas e feedback. Após as sugestões, o modelo passa por melhorias e segue para a próxima etapa: desenvolvimento.

\subsection{Desenvolvimento}

$\mathrm{Na}$ etapa de desenvolvimento, foram pesquisados modelos a serem utilizados para o desenvolvimento de Objetos de Aprendizagem (OAs) e Objetos de Aprendizagem Acessíveis (OAAs), considerando a ferramenta de desenvolvimento definida e os padrões e diretrizes de acessibilidade.

Conforme define Machado (2005), um Objeto de Aprendizagem (OA) é utilizado como recurso didático interativo, concebido como facilitador na construção do conhecimento para um determinado conteúdo. Por meio da utilização e interação com o OA, os alunos podem explorar o conteúdo e resolver os exercícios propostos de maneira prática e interativa. De acordo com Junqueira (2014), os objetos de aprendizagem são ferramentas ou recursos digitais que podem ser utilizados e reutilizados no suporte ao aprendizado.

O objetivo de desenvolver um OA com requisitos de acessibilidade parte das premissas de inclusão social de pessoas com deficiência, ou seja, é necessário garantir ao usuário independente ou não de necessidades especiais, a autonomia no acesso a informação no contexto de materiais digitais. (BRASIL, 2004). Desenvolver objetos de aprendizagem com requisitos de acessibilidade, permite aos alunos desenvolvedores, projetar e desenvolver os OAAs com um olhar inclusivo, envolve esforços e habilidades, visando reduzir os efeitos das limitações apresentadas nos ambientes e proporcionar uma maior igualdade aos PNEEs.

\subsubsection{Modelo de Desenvolvimento}

Foram pesquisados e selecionados alguns modelos, para o desenvolvimento dos objetos de aprendizagem, visando formalizar o processo. Neste sentido, as etapas precisam ser cumpridas em conformidade com o cronograma proposto, possibilitanto um gerenciamento de tempo, de recursos, de qualidade, de risco, de inicio e encerramento do projeto.

O trabalho de Barroso (2014), apresenta três modelos voltados para o desenvolvimento de objetos de aprendizagem, e todos eles incluem a fase de avaliação e 
V Congresso Brasileiro de Informática na Educação (CBIE 2016)

Anais do XXVII Simpósio Brasileiro de Informática na Educação (SBIE 2016)

a melhoria do conteúdo produzido. Os modelos abordados, podem ser observados na tabela 1 abaixo.

Tabela 1. Modelos de Desenvolvimento de OA, adaptado de Barroso (2014).

\begin{tabular}{|c|c|c|}
\hline \multicolumn{3}{|c|}{ MODELOS DE DESENVOLVIMENTO DE OBJETOS DE APRENDIZAGEM } \\
\hline RIVED & SOPHIA & ADDIE \\
\hline $\begin{array}{l}\text { Rede Interativa Virtual de Educação, } \\
\text { é um programa da Secretaria de } \\
\text { Educação a Distância (SEED) do } \\
\text { MEC, cujo objetivo é a produção de } \\
\text { OAs, e está dividido em } 6 \text { (seis) } \\
\text { fases. }\end{array}$ & $\begin{array}{l}\text { Visa auxiliar os alunos do curso de } \\
\text { Tecnologia em Análise e Desenvolvimento } \\
\text { de Sistemas (TADS), quanto à realização } \\
\text { de atividades e com conteúdo de apoio às } \\
\text { disciplinas, sendo o processo composto por } \\
\text { três fases. }\end{array}$ & $\begin{array}{l}\text { Sugerido por Mustaro et al (2007), } \\
\text { como guia para o desenvolvimento } \\
\text { de OAs, o qual é composto por } 5 \\
\text { (cinco) etapas. }\end{array}$ \\
\hline
\end{tabular}

Após a análise do modelo a ser utilizado como referência para o Desenvolvimento dos Objetos de Aprendizagem, foi escolhido o Modelo ADDIE (Análise, Projeto, Desenvolvimento, Implementação e Avaliação), em função da sua estrutura, do seu uso e dos resultados oriundos da sua aplicação. Neste sentido, a proposta é inserir em suas etapas do início ao final os requisitos de acessibilidade, visando atingir o objetivo do projeto. Desta forma, as atividades ficam mais claras para os alunos desenvolvedores e podem ser gerenciadas e monitoradas a suas entregas e cumprimentos.

De acordo com Mustaro et al (2007) o modelo é composto por cinco estágios, e considerando tais etapas, foram adaptadas as atividades deste projeto em cada uma delas, conforme pode-se observar:

- Análise: nesta etapa foram realizadas a definição e análise dos objetivos (principalmente educacionais) dos Objetos de Aprendizagem Acessíveis e dos requisitos de acessiblidade, padrões e diretrizes. Foram definidas as disciplinas, os conteúdos (unidade instrucional), objetivos, pré-requisitos, as mídias e os cenários. A turma foi dividida em equipes, sendo que cada equipe poderia ser composta por até 3 alunos.

- Projeto: esta fase acontece em paralelo à fase de Análise, ajustando assim os tópicos do projeto, antes da produção dos OAAs. Compreende os planos de construção do cenário do objeto, neste momento as equipes editam suas cenas usando a técnica de Storyboard. Também definem estratégias, interatividade, feedback, usabilidade, requisitos de acessibilidade, avaliação, entre outros;

- Desenvolvimento: nesta fase, após a definição da ferramenta, ocorre a criação dos OAs na ferramenta em conformidade com a análise e projeto especificados. Neste momento, algumas atividades são executadas, tais como: a edição, reunião e produção dos elementos multimídias, gerar ou conectar exercícios (avaliação), desenvolvimento de testes de usabilidade, programação de interação entre objetos, navegação (com base no modelo navegacional), feedback da avaliação para o aluno. Em paralelo, ocorrem reuniões com as equipes, visando monitorar as entregas e progressos, realizando críticas e sugerindo melhorias. A ferramenta de desenvolvimento definida em um primeiro momento foi o Sistema de autoria Visual Class (a UEA em seu investimento de capacitação docente, adquiriu a ferramenta e portanto possui a licença do software), o Visual Class é um software de autoria e gera os OAs para o padrão Web e XML. Em um segundo momento, a definição ficou em aberto, contando que as sugestões fossem 
V Congresso Brasileiro de Informática na Educação (CBIE 2016)

Anais do XXVII Simpósio Brasileiro de Informática na Educação (SBIE 2016)

avaliadas pelo professor coordenador, e caso não houvessem impedimentos de uso relacionado a licença e atendessem os padrões definidos, a ferramenta poderia ser aprovada e liberada o seu uso e implementação.

- Implementação: nesta fase deve ser definido o ROA (Repositório de Objetos de Aprendizagem), o qual será armazenado os OAs. Após a definição, realizar a inclusão dos metadados do objeto e testar a interface do ROA para com o objeto. O repositório definido foi o DSpace (2016), que é um software open source, um repositório usado por mais de 1000 organizações e instituições em todo o mundo para fornecer acesso duradouro aos recursos digitais, assim os objetos podem ser incluídos no Banco Internacional de Objetos Educacionais (BIOE) do MEC. O BIOE (2016) é um repositório que possui objetos educacionais de acesso público, em vários formatos e para todos os níveis de ensino. Os objetos podem ser acessados isoladamente ou em coleções (BIOE,2016).

- Avaliação: esta é a última fase, e compreende todo o processo de desenvolvimento. Nesta etapa, o objeto de aprendizagem é avaliado quanto ao conteúdo e pelos seus itens de ensino e aprendizado. Essa avaliação, deve ocorrer com o auxílio de alunos e professores. As equipes realizam a apresentação dos objetos produzidos. Desta forma, com as críticas e sugestões realizadas, os erros e falhas são minimizados.

\subsubsection{Diretrizes, Padrões e Acessibilidade}

Segundo Macedo (2010) a adoção de padrões globais na criação, indexação, e tecnologia de objetos de aprendizagem é crucial para assegurar a reutilização e interoperabilidade e qualidade dos objetos de aprendizagem.

O SCORM é o padrão mais adotado por todos os principais provedores de ferramentas de autoria e sistemas de gerenciamento de aprendizagem (LMS) (MOORE e KEARSLEY, 2007). A W3C é o órgão responsável pelas especificações como XML, HTML e RDF. Suas especificações constituem os padrões industriais. Cria especificações de mais baixo nível que o IMS. Por exemplo, o IMS usa XML como linguagem para representação de metadados, perfis e outras informações estruturadas (IMS GLC, 2010).

A acessibilidade à Web é parte integrante do Projeto Brasileiro de Inclusão Digital para as pessoas portadoras de necessidades especiais. O artigo 47 do Decreto 5.296 exige a acessibilidade nos portais e sítios eletrônicos da administração pública na internet, para o uso das pessoas portadoras de deficiência visual, garantindo-lhes o pleno acesso às informações disponíveis (MACEDO,2010).

\subsection{Validação}

Nesta etapa, são realizadas as avaliações da aprendizagem e da usabilidade dos OAAs. Num primeiro momento, as avaliações são feitas por meio da aplicação de questionários, onde os avaliadores são especialistas da area pedagógica (ensino e aprendizagem), de usabilidade (usabilidade) e de informática na educação (acessibilidade). Os resultados, assim como críticas e sugestões ouriundas desta seção, são válidas para a melhoria final dos objetos antes da aplicação e são comentados na próxima seção de resultados e discussão. 
V Congresso Brasileiro de Informática na Educação (CBIE 2016)

Anais do XXVII Simpósio Brasileiro de Informática na Educação (SBIE 2016)

\section{Resultados e Discussões}

Fundamentado nos padrões, modelos e diretrizes de acessibilidade definidos em Macedo (2010), e questões de design e usabilidade descritos em Preece (2002), observou-se que os objetos de aprendizagem acessíveis desenvolvidos, em grande maioria correspondem as expectativas e atendem aos requisitos e objetivos definidos. Neste sentido, é importante salientar a importância do cumprimento, monitoramento e gerenciamento das etapas de planejamento, desenvolvimento e validação.

Na etapa de planejamento, o plano de aula foi desenvolvido em conformidade com o plano de ensino repassado para cada equipe, onde foram definidos: objetivos, recursos, tempo, avaliação e unidade instrucional. A abordagem pedagógica foi definida, os fundamentos epistemológicos foram pesquisados em Darsie (1999). O design pedagógico foi desenvolvido com auxilio da técnica de Storyboard, permitindo as equipes rascunhar suas produções por meio de um desenho preliminar.

$\mathrm{Na}$ etapa de desenvolvimento, foi utilizado o software de autoria Visual Class o qual permitiu a implementação dos desenhos e cenas criados em conformidade com os padrões e diretrizes de acessibilidade propostos, bem como o uso do padrão XML e SCORM. As equipes foram bastante criativas e atuantes, cumprindo os prazos estabelecidos no cronograma.

$\mathrm{Na}$ etapa de validação foram realizadas as avaliações: da aprendizagem, de ensino, de usabilidade e de acessibilidade com os especialistas. Foram desenvolvidos quatro tipos de questionários e suas respectivas questões foram elaboradas, considerando itens relacionados a cada tipo de avaliação, em consonância com as metodologias de avaliação voltadas para objetos de aprendizagem. A estratégia utilizada para aplicação foi determinar 30 a 40 minutos para a apresentação dos OAs e que poderiam haver orientações durante o processo.

A Avaliação de Aprendizagem: sugere que o aluno já tenha visto o conteúdo antes da aplicação do objeto de aprendizagem e que o professor possa ser atuante durante o processo. Neste contexto, verifica-se os conhecimentos que os alunos possuem sobre os OAs e observa-se o que os alunos aprenderam com ele. A tabela 2 abaixo, demonstra os resultados obtidos na avaliação de aprendizagem.

Tabela 2. Questões e percentual gerado na Avaliação de Aprendizagem.

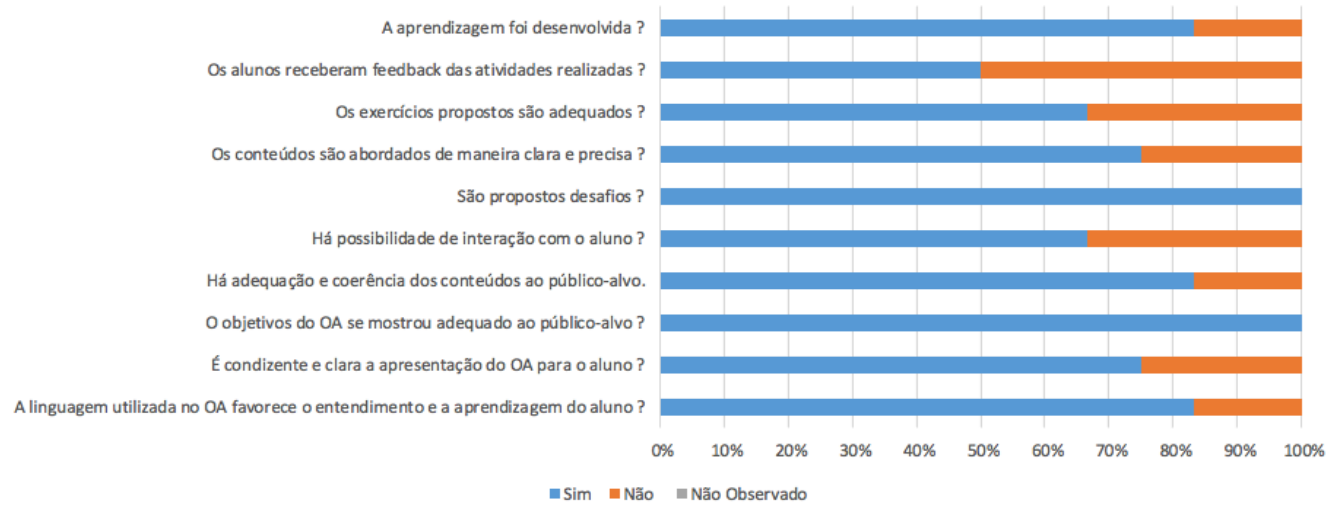

$\mathrm{Na}$ análise foi avaliada que os OAAs desenvolvidos em $82 \%$ a aprendizagem foi desenvolvida, e a linguagem utilizada favorece o entendimento por parte do aluno e afirmam haver adequação e coerência dos conteúdos ao público-alvo. Em 100\% são 
V Congresso Brasileiro de Informática na Educação (CBIE 2016)

Anais do XXVII Simpósio Brasileiro de Informática na Educação (SBIE 2016)

propostos desafios (atividades) e afirmam que os objetivos dos OAAs se mostraram adequados ao público-alvo. Sendo preciso uma melhoria significativa na interação e no feedback das atividades desenvolvidas.

A Avaliação de Ensino: refere-se à adequação dos objetivos e dos conteúdos, reuso e qualidade do material desenvolvido. A tabela 3 abaixo, demonstra os resultados obtidos na avaliação do ensino.

\section{Tabela3. Questões e percentual gerado na Avaliação do Ensino.}

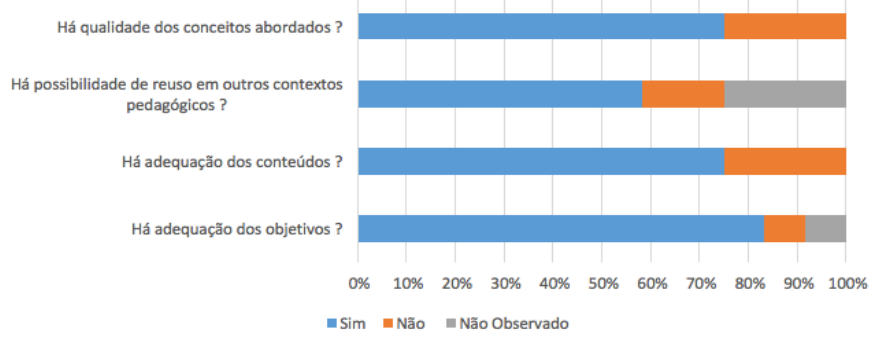

$\mathrm{Na}$ análise constatou-se que $82 \%$ dos objetos estão adequados aos objetivos definidos, $74 \%$ apresentam qualidade dos conceitos abordados e adequação dos conteúdos e $58 \%$ expressam que há possibilidade de reuso em outros contextos pedagógicos.

A Avaliação de Usabilidade: permite avaliar a qualidade de um sistema com relação a fatores que os projetistas definem como sendo prioritários ao sistema. Os itens de usabilidade definidos por Preece et al (2002) foram utilizados nesta avaliação. A tabela 4 a seguir, apresenta os resultados obtidos na avaliação de usabilidade.

Tabela 4. Questões e percentual gerado na Avaliação de Usabilidade.

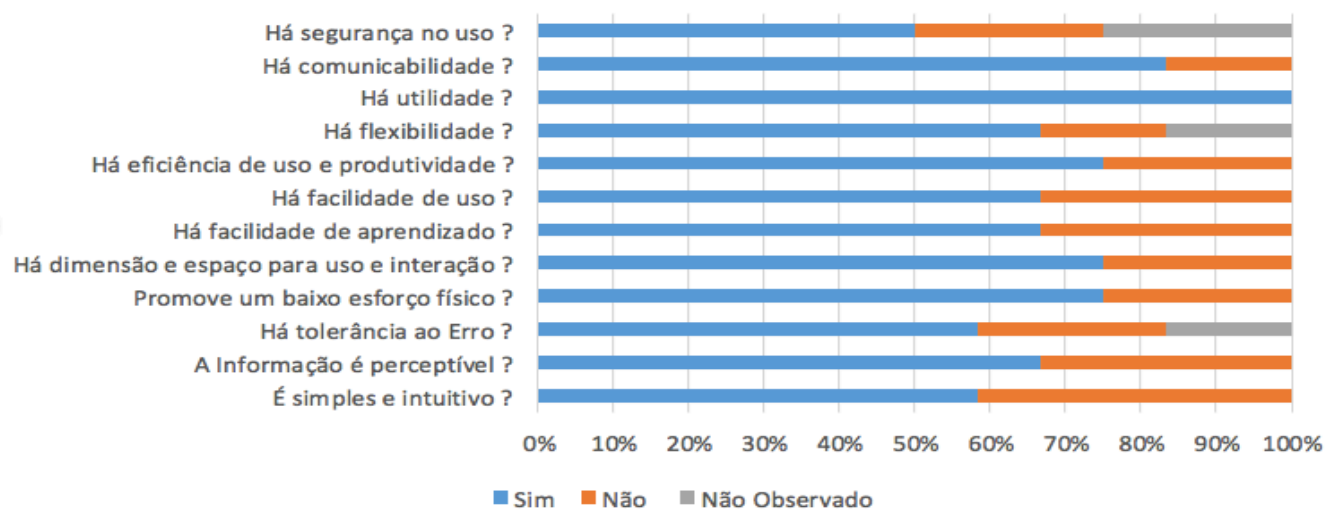

$\mathrm{Na}$ analise realizada $100 \%$ relataram a utilidade dos OAs, destes $82 \%$ apontaram que há comunicabilidade; $75 \%$ relataram que há eficiência do uso e produtividade, que há dimensão e espaço para o uso e interação e que promovem um baixo esforço físico. Sendo que $58 \%$ afirmaram que há tolerância ao erro e que demonstram ser simples e intuitivo.

A Avaliação de Acessibilidade: permite avaliar práticas recomendadas aos desenvolvedores, fornecedores de conteúdo e educadores envolvidos na criação de produtos para aprendizagem, produção de aplicativos de softwares e conteúdos acessíveis, cujos princípios para acessibilidade são apresentados pelo IMS (2002). A tabela 5 a seguir, apresenta os resultados obtidos na avaliação de acessibilidade. 
V Congresso Brasileiro de Informática na Educação (CBIE 2016)

Anais do XXVII Simpósio Brasileiro de Informática na Educação (SBIE 2016)

Tabela 5. Questões e percentual gerado na Avaliação de Acessibilidade.

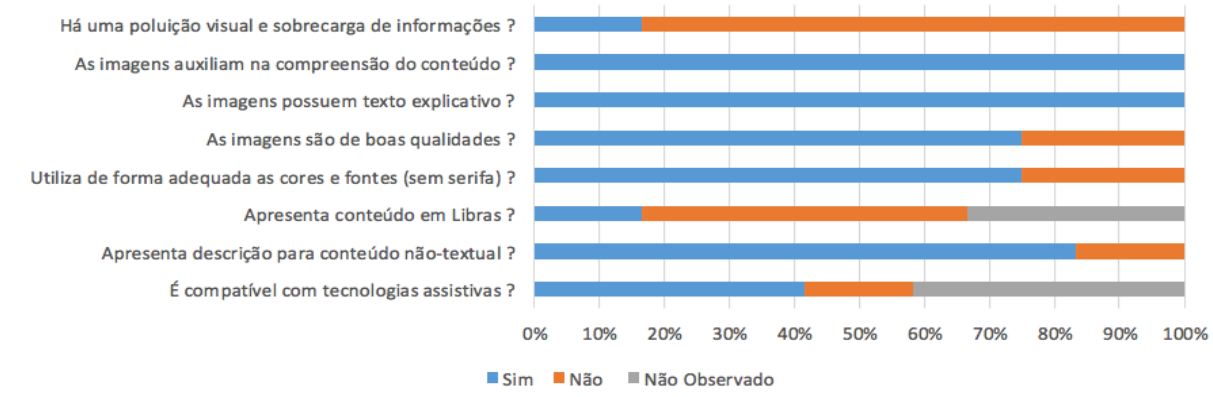

No processo de análise de acessibilidade $100 \%$ dos avaliadores relatam que as imagens auxiliam na comprenssão do conteúdo e que possuem texto explicativo. Afirmam $82 \%$ que os OAAs apresentam descrição para o conteúdo não-textual; outros $75 \%$ relatam que as imagens são de boas qualidades e utiliza de forma adequada as cores e fontes (sem serifa). Apenas $42 \%$ é compatível com as tecnologias assistivas e $16 \%$ apresentam conteúdo em libras e apresentam poluição visual e sobrecarga de informações. O percentual apresentado ao item não observado referem-se as tecnologias assitivas e ao conteúdo em libras, isso reflete no desconhecimento do uso de tais tecnologias.

As críticas e recomendações realizadas pelos especialistas refletem nos itens de usabilidade e acessibilidade, que se trabalhadas em paralelo permitirão aos acadêmicos PNEEs e não portadores, a terem acesso a um recurso educacional rico em conteúdo e que contribuirá significativamente para o processo de ensino e aprendizagem. As avaliações realizadas são feedback importantes para os desenvolvedores, com intuito de mensurar e identificar problemas ou limitações a serem solucionados antes de sua finalização e entrega.

\section{Conclusão e Trabalhos futuros}

O modelo proposto tem como objetivo direcionar e motivar docentes da área da computação ou afins, a seguir um processo para elaboração de recursos educacionais voltados para PNEEs, promovendo a inclusão social e digital, dada a escassez de recursos voltados para este público-alvo.

O projeto desenvolvido trouxe uma contribuição e experiência significativa para os alunos do curso, que pretendem implementar novos objetos na sua disciplina de estágio, visando colaborar com as escolas as quais estão estagiando. As etapas de planejamento, desenvolvimento e validação, que constituem o modelo, quando seguidas, gerenciadas, monitoradas e mediadas pelo professor permitem o sucesso do projeto. O projeto permite que modelos, ferramentas e parâmetros especificos sejam utilizados em conformidade com os objetivos e estratégias definidas para o desenvolvimento dos OAAs.

$\mathrm{Na}$ fase de planejamento constatou-se que material pedagógico inserido nas atividades está condizente com os objetivos e adequado à faixa etária e público-alvo da proposta. Na fase de desenvolvimento percebeu-se algumas limitações em relação ao software de autoria Visual Class, pois quando requerido algumas funcionalidades, resultou na utilização de novas estratégias e adequações de conteúdo e formatos de avaliações. Na fase de validação, constatou-se a relevância da aplicação dos 
V Congresso Brasileiro de Informática na Educação (CBIE 2016)

Anais do XXVII Simpósio Brasileiro de Informática na Educação (SBIE 2016)

questionários e do envolvimento dos especialistas, que permitiu que os OAAs, fossem avaliados com profissionalismo. As qualidades e limitações apresentadas foram consideradas, visando a melhoria do processo, e o uso de ferramentas especificas dependendo do tipo e nível de deficiência apresentado. Os dados quantitativos gerados serviram como referência para as melhorias dos OAAs. Os problemas ou limitações identificados foram solucionados antes de sua finalização. Portanto, este modelo se utilizado adequadamente, permitirá o desenvolvimento dos OAAs, desde o inicio até o encerramento do projeto, resultando no seu sucesso.

De acordo com Becker (2014) a inclusão sócio-digital refere-se a ações de inserção no cunho tecnológico e político que promovam o desenvolvimento de tecnologias que ampliem a acessibilidade de pessoas com deficiências. Desta forma, como trabalhos futuros espera-se aplicar os Objetos de Aprendizagem Acessíveis produzidos, em dois ambientes de sala de aula, cuja disciplina envolva o conteúdo (unidade instrucional) desenvolvido. No primeiro ambiente, que seja uma turma que haja pelo menos um aluno PNEEs, e no segundo ambiente com uma turma que não possui alunos PNEEs, verificando assim se a proposta atende aos dois públicos.

\section{Referências}

ABC. Associação Brasileira de Cinematografia. (2016), Storyboard. Disponível em: http://www.abcine.org.br/servicos/?id=158\&/storyboard. Acessada em: 04/03/2016.

Araújo, Also De, Rr De Brito, and Ap Da Silva. (2013), "Softwares Para Educação Inclusiva: Uma Revisão Sistemática No Contexto de SBIE E WIE.” ... de Informática na Educação Cbie (2013): 507-515. Web.

BIOE. Banco Internacional de Objetos Educacionais. (2016). Disponível em: http://www.dspace.org. Acessado em: Fevereiro de 2016.

Barroso, Giovanni Cordeiro et al. (2014), "Um Modelo Para a Produção de Objetos de Aprendizagem Acessíveis: Modelagem E Análise Por Redes de Petri Coloridas.” Cbie (2014): 1143-1152. Print.

Brasil, Decreto-lei no 5.296, de 2 de dezembro de 2004. (2004). Diário Oficial da República Federativa do Brasil, Poder Executivo, Brasília, DF, 03 dez. 2004.

Becker, Deisi et al. "O Uso de Objetos de Aprendizagem Com Alunos Surdos No Ensino Superior." Cbie (2014): 832-836.

Darsie, M. M. P. (1999). "Perspectivas Epistemológicas e suas Implicações no Processo de Ensino e de Aprendizagem”. Cuiabá, Uniciências, v3: 9-21.

DSpace. Repositório de Objetos de Aprendizagem. (2016). Disponível em: http://www.dspace.org. Acessado em: Fevereiro de 2016.

IMS GLC. IMS Guidelines for Developing Accessible Learning Applications V.1 White Paper. IMS Global Learning Consortium Inc., 27 junho 2002. Disponível em: <http://www.imsglobal.org/accessibility/>. Acesso em: 02 fevereiro 2016.

Junqueira, Rossana De Paula. "Repositórios de Objetos de Aprendizagem: Uma Análise Comparativa Com Ênfase No Reúso de Conteúdos.” Cbie (2014): 988-992.

Lei N. ${ }^{\circ} 7.853$, de 24 de outubro de 1989. Disponível em: http://www.planalto.gov.br/ccivil_03/leis/17853.htm. Acessado em: Abril de 2016.

Macedo, C. M. S. (2010). Diretrizes para criação de objetos de aprendizagem acessíveis. Tese de Doutorado, Programa de Pós-graduação em Engenharia e Gestão do Conhecimento, Mídia e Conhecimento, Universidade Federal de Santa Catarina Florianópolis, SC.

Moore, M. G.; Kearsley, G. Educação a Distância - Uma Visão Integrada. Tradução de Roberto Galman. 1. ed. São Paulo: Thomson Learning, v. 1, 2007. 398 p. ISBN 978-85-221-0576-2.

Mustaro, P. N., Silveira, I. F., Omar, N. e Stump, S. M. D. (2007), "Structure of Storyboard for Interactive Learning Objects Development", Learning Objects: and instructional design, Koohang, A. and Harman, K., Santa Rosa, Informing Science Press, p.253-279.

Preece, J.; Rogers, Y.; Sharp, E. (2002) Interaction Design: Beyond Human-computer Interaction. New York, NY: John Wiley \& Sons. 2002.

StoryboardThat. (2016). Disponível em: http://www.storyboardthat.com. Acessada em: 04/03/2016. 